\title{
A Sidon-type condition on set systems
}

\author{
Peter J. Dukes* and Jane Wodlinger*
}

\begin{abstract}
Consider families of $k$-subsets (or blocks) on a ground set of size $v$. Recall that if all $t$-subsets occur with the same frequency $\lambda$, one obtains a $t$-design with index $\lambda$. On the other hand, if all $t$-subsets occur with different frequencies, such a family has been called (by Sarvate and others) a $t$-adesign. An elementary observation shows that such families always exist for $v>k \geq t$. Here, we study the smallest possible maximum frequency $\mu=\mu(t, k, v)$.

The exact value of $\mu$ is noted for $t=1$ and an upper bound (best possible up to a constant multiple) is obtained for $t=2$ using PBD closure. Weaker, yet still reasonable, asymptotic bounds on $\mu$ for higher $t$ follow from a probabilistic argument. Some connections are made with the famous Sidon problem of additive number theory.
\end{abstract}

\section{Introduction}

Given a family (which may contain repetition) $\mathcal{A}$ of subsets of a ground set $X$, the frequency of a set $T \subset X$ is the number of elements of $\mathcal{A}$ (counting multiplicity) which contain $T$.

Let $v \geq k \geq t$ be nonnegative integers. A $t$-design, or $S_{\lambda}(t, k, v)$, is a pair $(V, \mathcal{B})$ where $\mathcal{B}$ is a family of $k$-subsets of $V$ such that every $t$-subset has the same frequency $\lambda$. Typically, $V$ is called a set of points, $\mathcal{B}$ are the blocks, $t$ is the strength (reflecting that $t$-designs are also $i$-designs for $i \leq t$ ) and $\lambda$ is the index. Repeated blocks are normally permitted in the definition.

There are 'divisibility' restrictions on the parameters $v, k, t, \lambda$ and beyond that very little is known in general about the existence of $S_{\lambda}(t, k, v)$. There are some trivial cases, such as $t=0, t=k$, or $k=v$, and some mildly interesting ones: $\lambda=t=1$ leads to uniform partitions; $\lambda=\left(\begin{array}{l}v-t \\ k-t\end{array}\right)$ is realized via the complete $k$-uniform hypergraph of order $v$. For $t=2$ and fixed $k$ there is a rich and deep asymptotic existence theory due to R.M. Wilson; see [5]. Spherical geometries and Hadamard matrices lead to some examples for $t=3$.

\footnotetext{
${ }^{*}$ This research is supported by NSERC.
} 
In [4], Sarvate and Beam consider an interesting twist on the definition. A $t$-adesign is defined as a pair $(V, \mathcal{A})$, where $V$ is a ground set of $v$ points and $\mathcal{A}$ is a collection of blocks of size $k$, satisfying the condition that every $t$-subset of points has a different frequency.

Here, we abbreviate a $t$-adesign with $A(t, k, v)$. It is easy to see that such families always exist for integers $v>k \geq t \geq 1$ : simply assign multiplicities to $\left(\begin{array}{l}V \\ k\end{array}\right)$ according to different powers of two.

This begs a more intricate question. Let $\mu(t, k, v)$ denote the smallest maximum frequency, taken over all adesigns $A(t, k, v)$. The main question motivating this article is the following.

Problem 1.1. Given $t, k, v$, determine (or bound) $\mu(t, k, v)$.

In most of the previous investigations on adesigns, the cases of interest have been for $t=2$ and when the different pairwise frequencies are $1,2, \ldots,\left(\begin{array}{l}v \\ 2\end{array}\right)$. It should be noted that here we allow zero as a frequency, although, if desired, it is not hard to bump up all frequencies to be positive.

From the definitions and easy observations above, we have

$$
\left(\begin{array}{l}
v \\
t
\end{array}\right)-1 \leq \mu(t, k, v)<2^{\left(\begin{array}{l}
v \\
k
\end{array}\right)}
$$

However, the basic upper bound in (1) is unsatisfactory, at least asymptotically in $v$. Our main goal is a substantial reduction of the upper bound (to something independent of $k$ ).

Theorem 1.2. For $k>2 t+2$ and sufficiently large $v$,

$$
\mu(t, k, v) \leq 16 t v^{2 t+2} \log v
$$

The constant is surely not best possible; however, we are content until more is known about the exponent.

We can do much better when $t \leq 2$. For $t=1$, an elementary argument gives the exact value of $\mu$. And for $t=2$, Wilson's theory of PBD closure reduces the upper bound on $\mu$ to a constant multiple of its lower bound.

Theorem 1.3. For positive integers $v>k$,

$$
\mu(1, k, v)= \begin{cases}v-1 & \text { if } 2 k \leq v \text { and }\left(\begin{array}{l}
v \\
2
\end{array}\right) \equiv 0 \quad(\bmod k) \\
v & \text { if } 2 k \leq v \text { and }\left(\begin{array}{l}
v \\
2
\end{array}\right) \neq 0 \quad(\bmod k) \\
\left\lceil\frac{1}{v-k}\left(\begin{array}{l}
v \\
2
\end{array}\right)\right] & \text { if } 2 k>v\end{cases}
$$


Theorem 1.4. There is a constant $C=C(k)$ such that $\mu(2, k, v) \leq C v^{2}$.

The proof of Theorem 1.2 follows a probabilistic argument and occurs in Section 2. The proofs of Theorems 1.3 and 1.4 are given in Section 3.

Before beginning our detailed investigations, we should mention some connections with a central topic in additive combinatorics. Briefly, a Sidon sequence (or Golomb ruler) is a list of positive integers whose pairwise sums are all distinct, up to swapping summands. More generally, a $B_{r}$-sequence or Sidon sequence of order $r$ has the property that all its $r$-wise sums are distinct. It is known (see [3]) that the largest cardinality $F_{r}(n)$ of a Sidon sequence of order $r$ contained in $[n]$ satisfies

$$
n^{1 / r}(1-o(1)) \leq F_{r}(n) \leq C(r) n^{1 / r} .
$$

Now consider an adesign $A(t, v-1, v)$, where $V$ is the ground set of size $v=k+1$. Assign multiplicity $f(x)$, chosen from a Sidon sequence of order $t$, to the 'co-singleton' set $V \backslash\{x\}, x \in V$. The inherited weight on a $t$-subset $T$ is $\sum_{x \notin T} f(x)$. By construction, this takes distinct values on all $t$-subsets. From this and (2), we see that $\mu(t, v-1, v) \leq C v^{t}$, which is best possible up to a constant multiple. However, it is also clear that the exact determination of $\mu$, even in the case $v=k+1$, is as difficult as the Sidon problem.

\section{The general bound}

We prove Theorem 1.2 by employing $B_{r}$-sequences along the lines of the discussion concluding Section 1. But here, a probabilistic selection is needed to control the upper bound on $\mu$.

Proof of Theorem 1.2. Assume $t>1$, appealing to Theorem 1.3. Suppose first that $v$ is a prime power. Bose and Chowla [1] construct a $B_{t}$-sequence of size $v$ in $\left[v^{t}\right]$. Let $V$ be such a set of integers and consider the family $\mathcal{B}$ of all $k$-subsets of $V$, where a $k$-set $K$ is taken with multiplicity

$$
f(K)=\sum_{m \in V \backslash K} m .
$$

Then the frequency of a $t$-subset $T$ in $\mathcal{B}$ is

$$
f(T)=\sum_{K \supseteq T,|K|=k} f(K)=\left(\begin{array}{c}
v-t-1 \\
k-t
\end{array}\right) \sum_{m \in V \backslash T} m .
$$


By choice of $V$, these are all distinct frequencies. Observe that $\sum_{m \in V \backslash T} m<$ $v^{t}(v-t)$, so that $f(T)$ is at most a polynomial of order $v^{k+1}$.

Consider next a family $\mathcal{A}=\mathcal{A}(p)$ consisting of each element of $\mathcal{B}$ chosen independently with probability $p$. We claim there is some $p$ guaranteeing an adesign $A(t, k, v)$ of the required form.

Let $f_{\mathcal{A}}(T)$ denote the frequency of $T$ in $\mathcal{A}$. This is a sum of $f(T)$ independent binomial random variables $X_{i}$, one for each $k$-set in $\mathcal{B}$ containing $T$. So $f_{\mathcal{A}}(T)$ has expected value $\mu=p f(T)$ by linearity.

Now let's invoke a (weak but tidy) two-sided Chernoff bound of the form

$$
\mathbb{P}\left[\left|\sum X_{i}-\mu\right|>2 \sqrt{\mu \log 1 / \epsilon}\right]<\epsilon,
$$

which holds for $\epsilon>\exp (-\mu / 4)$. Taking $\epsilon=\left(\begin{array}{c}v \\ t\end{array}\right)^{-1}$, we conclude that there exists (with positive probability) a family $\mathcal{A}$ such that

$$
\left|f_{\mathcal{A}}(T)-p f(T)\right|<\sigma(T)
$$

for every $t$-set $T$, where $\sigma(T):=2 \sqrt{p f(T) \log \left(\begin{array}{c}v \\ t\end{array}\right)}$, and for each $p$ with $p f(T)>4 \log \left(\begin{array}{l}v \\ t\end{array}\right)$.

It remains to check that frequencies $f_{\mathcal{A}}(T)$ remain distinct and appropriately bounded for some choice of $p$. By (3) and (4), we have distinct frequencies provided that

$$
2 \sigma(T)<p\left(\begin{array}{c}
v-t-1 \\
k-t
\end{array}\right)
$$

Using the definition of $\sigma(T)$ and $\sum_{m \notin T} m<v^{t}(v-t)$, it suffices to have

$$
16 v^{t}(v-t) \log \left(\begin{array}{l}
v \\
t
\end{array}\right)<p\left(\begin{array}{c}
v-t-1 \\
k-t
\end{array}\right) .
$$

The right side of (5) grows faster than the left for $k \geq 2 t+2$; hence, for sufficiently large $v$, we can choose $p=16 v^{t+1} \log \left(\begin{array}{c}v \\ t\end{array}\right) /\left(\begin{array}{c}v-t-1 \\ k-t\end{array}\right)<1$ (easily permitting application of the Chernoff bound above).

For such a choice, we have

$$
\max _{T} f_{\mathcal{A}}(T)<p f(T)+\sigma(T)<\left(16 v^{2 t+2}+8 v^{t+1}\right) \log \left(\begin{array}{l}
v \\
t
\end{array}\right) .
$$

The bound $\log \left(\begin{array}{l}v \\ t\end{array}\right) \leq t \log v-\log t$ ! leaves enough room to eliminate the lower-order term and imply the stated bound. 
Finally, if $v$ is not a prime power, we can simply apply the above argument to a prime $v^{\prime} \leq v+o(v)$ to obtain asymptotically the same result.

\section{The cases $t=1$ and $t=2$}

When $t=1$, we simply demand that every point is in a different number of blocks. A complete characterization is possible here, following a technique known to Sarvate and Beam in early investigations. To the best of our knowledge, though, Theorem 1.3 has not been worked out for general $v$ and $k$.

The proof strategy is as follows. Suppose $f(1)<\cdots<f(v)$ are desired pointwise frequencies whose sum $F$ is divisible by $k$. Set up $b=F / k$ blocks, and place element ' 1 ' in the first $f(1)$ blocks, element ' 2 ' in the next $f(2)$ blocks, and so on, with blocks identified modulo $b$. In other words, the $i$ th block contains those elements $x$ such that

$$
\sum_{1 \leq y<x} f(y)<b q+i \leq \sum_{1 \leq y \leq x} f(y)
$$

for some integer $q \in\{0,1, \ldots, k-1\}$. Care must be taken that the maximum frequency $f(v)$ does not exceed $b$, the number of blocks. Ideally, the frequencies are chosen to be consecutive, or almost consecutive, integers.

Proof of Theorem 1.3. We apply the above construction using a run of (almost) consecutive prescribed frequencies. There is a division into two main cases.

Case $1.2 k \leq v$. Suppose first that $k \mid\left(\begin{array}{l}v \\ 2\end{array}\right)$. Fill $b=\left(\begin{array}{l}v \\ 2\end{array}\right) / k$ blocks with pointwise frequencies $0,1, \ldots, v-1$. Note that $b \geq v-1$ follows from the assumption $2 k \leq v$. On the other hand, if $\left(\begin{array}{l}v \\ 2\end{array}\right)=b k-r, 0<r<k$, use $b$ blocks with frequencies $0,1, \ldots, v-r-1, v-r+1, \ldots, v$. One has sum of frequencies $b k=\left(\begin{array}{c}v+1 \\ 2\end{array}\right)-(v-r)=\left(\begin{array}{l}v \\ 2\end{array}\right)+r$, as required. In either sub-case, the smallest possible maximum frequency is realized and we have

$$
\mu(1, k, v)=\left\{\begin{array}{lll}
v-1 & \text { if }\left(\begin{array}{l}
v \\
2
\end{array}\right) \equiv 0 \quad(\bmod k), \\
v & \text { if }\left(\begin{array}{l}
v \\
2
\end{array}\right) \neq 0 \quad(\bmod k) .
\end{array}\right.
$$

Case 2. $2 k>v$. We first show that the given value $\left\lceil\frac{1}{v-k}\left(\begin{array}{l}v \\ 2\end{array}\right)\right\rceil$ is a lower bound on $\mu(1, k, v)$. Suppose $m$ is the maximum frequency in an adesign $A(1, k, v)$. Then

$$
m k \leq b k \leq(m-v+1)+\cdots+(m-1)+m=m v-\left(\begin{array}{l}
v \\
2
\end{array}\right) .
$$


In other words, $m$ is an integer with $m(v-k) \geq\left(\begin{array}{l}v \\ 2\end{array}\right)$ and the lower bound follows. Conversely, we must realize the given value $\mu:=\left\lceil\frac{1}{v-k}\left(\begin{array}{c}v \\ 2\end{array}\right)\right\rceil$ as the maximum frequency in an adesign $A(1, k, v)$. Put $b k=\mu v-\left(\begin{array}{c}v \\ 2\end{array}\right)-r$, for some positive integer $b$ and $0 \leq r<k$. Again, use the strategy preceding the statement of the theorem, with $b=\frac{1}{k}\left(\mu v-\left(\begin{array}{l}v \\ 2\end{array}\right)-r\right)$ blocks and frequencies

$$
\mu-v, \ldots, \mu-v-r-1, \mu-v-r+1, \ldots, \mu .
$$

It remains to check that $\mu \leq b$. However, this follows easily since $\mu$ is the least integer with $\mu(v-k) \geq\left(\begin{array}{l}v \\ 2\end{array}\right)$. Therefore, $\mu k \leq \mu v-\left(\begin{array}{l}v \\ 2\end{array}\right)$. On the other hand, $b$ is the greatest integer so that $b k \leq \mu v-\left(\begin{array}{l}v \\ 2\end{array}\right)$.

We turn now to adesigns with $t=2$. An important tool here is 'PBD closure,' which we briefly outline. Let $K$ be a set of positive integers, each at least two. A pairwise balanced design $\operatorname{PBD}(v, K)$ is a set of $v$ points, together with a set of blocks whose sizes are in $K$, having the property that every unordered pair of different points is contained in exactly one block. Wilson's theorem [5] asserts that the necessary 'global' and 'local' divisibility conditions on $v$ given $K$ are asymptotically sufficient for the existence of $\operatorname{PBD}(v, K)$.

A key observation for the proof of Wilson's theorem is the 'breaking up blocks' construction: a block, say of size $u$, of a PBD can be replaced with the family of blocks of a PBD on $u$ points. In particular, if there exists a $\operatorname{PBD}(v, K)$ and an $S_{\lambda}(2, k, u)$ for every $u \in K$, then there exists an $S_{\lambda}(2, k, v)$.

It was observed in [2] that adesigns actually obey a similar recursion. The basic idea is to place adesigns (instead of designs) on the blocks of a PBD. However, each such adesign needs to be accompanied with a block design on those points with sufficiently large $\lambda$ so as to 'spread out' the pairwise frequencies. When restated using $\mu$, one obtains the following result.

Lemma 3.1. Suppose there exists a $P B D(v, K)$ with $b$ blocks having sizes $u_{1}, u_{2}, \ldots, u_{b}$. Put $M_{0}=0$ and for $0<i \leq b$,

$$
M_{i}=\min \left\{\lambda \geq M_{i-1}: \exists S_{\lambda}\left(2, k, u_{i}\right)\right\}+\mu\left(2, k, u_{i}\right) .
$$

Then $\mu(2, k, v) \leq M_{b}$.

Remark. The minimum in (6) is well defined; more generally, $S_{\lambda}(t, k, v)$ exists for a smallest positive integer $\lambda=\lambda_{\min }(v, k) \leq\left(\begin{array}{l}v-t \\ k-t\end{array}\right)$, and such designs can be repeated with arbitrary multiplicity.

We are now ready to prove the quadratic upper bound on $\mu(2, k, v)$. 
Proof of Theorem 1.4. For $v$ large and $K=\{k+1, k+2, k+3\}$, apply Lemma 3.1 to a $\operatorname{PBD}(v, K)$. Note that such $\operatorname{PBDs}$ exist for all sufficiently large integers $v$. This follows easily from Wilson's theorem since the three consecutive block sizes lead to no 'divisibility' restrictions on $v$ (globally, $\operatorname{gcd}\{k(k+1),(k+1)(k+2),(k+2)(k+3)\}=2$ always divides $\left(\begin{array}{l}v \\ 2\end{array}\right) ;$ locally, $\operatorname{gcd}\{k, k+1, k+2\}=1$ divides $v-1)$.

Put $m=\max \{\mu(2, k, k+j): j=1,2,3\}$ and $l=\max \left\{\lambda_{\min }(k+j, k):\right.$ $j=1,2,3\}$. Observe that $l$ and $m$ depend only on $k$. Also, observe that the number of blocks $b$ of a $\operatorname{PBD}(v, K)$ satisfies $b \leq\left(\begin{array}{c}v \\ 2\end{array}\right) /\left(\begin{array}{c}k+1 \\ 2\end{array}\right)$, since $k+1$ is the smallest block size. Combining these facts, it follows that

$$
\mu(2, k, v) \leq \operatorname{lmb} \leq C(k)\left(\begin{array}{l}
v \\
2
\end{array}\right) .
$$

\section{Discussion}

There is another noteworthy construction of $t$-adesigns by combining copies of systems which are nearly designs. The general idea to work from a family $\widehat{\mathcal{B}}_{T}$ of $k$-subsets such that one preferred $t$-subset $T$ has frequency $\lambda_{1}$ and all other $t$-subsets have frequency $\lambda_{2}<\lambda_{1}$. (Such families can be found, for instance, via a linear algebraic argument upon 'clearing denominators.') Then, take copies of $\widehat{\mathcal{B}}_{T}$ with distinct multiplicities over each $T \in\left(\begin{array}{c}V \\ t\end{array}\right)$. The crude bound obtained in this way is $\mu(t, k, v) \leq C_{1} \lambda_{1} v^{t}+C_{2} \lambda_{2} v^{2 t}$. However, we presently see no way of keeping $\lambda_{2}$ small enough in general. This would be an interesting problem in its own right. When such families $\widehat{\mathcal{B}}_{T}$ exist with reasonable $\lambda_{2}$, it is possible to improve Theorem 1.2.

The remaining work for $t=2$ essentially amounts to a reduction in the multiplicative constant in Theorem 1.4. There are some ideas which seem promising in this direction. For instance, $\mu(2,3, v)$ was completely determined in [2] using a blend of PBD closure, group divisible designs, and a variation on 'anti-magic cubes.' The latter concerns a neat side-problem: place nonnegative integers in the cells of the cube $[n]^{3}$ so that the $3 n^{2}$ line sums are distinct and with maximum value as small as possible. Interesting constructions yielding line sums $\left\{0,1, \ldots, 3 n^{2}-1\right\}$ were found for $n=2,3,5,7$, and products of these values.

Returning to $\mu(2,3, v)$, a slightly technical argument shows that the maximum frequency for triples versus pairs in an adesign is best possible.

Theorem 4.1 ([2]). For all $v>3$,

$$
\mu(2,3, v)=\left\{\begin{array}{l}
\left(\begin{array}{l}
v \\
2
\end{array}\right), \text { if } v=4 \text { or } v \equiv 2 \quad(\bmod 3), \\
\left(\begin{array}{l}
v \\
2
\end{array}\right)-1, \text { otherwise }
\end{array}\right.
$$


We omit further analysis of $\mu(2, k, v)$ for $k>3$ until better general constructions surface for small $v$ relative to $k$. For fixed $k$, the complete determination of $\mu(2, k, v)$ can probably be reduced to a finite problem. Quite possibly $\mu(2, k, v)=\left(\begin{array}{l}v \\ 2\end{array}\right)-1+o(v)$ for each $k$.

\section{Acknowledgement}

The authors are indebted to the anonymous referee who suggested the probabilistic method, yielding the present form of Theorem 1.2.

\section{References}

[1] R. C. Bose and S. Chowla (1962/1963). Theorems in the additive theory of numbers. Comment. Math. Helv. 37, 141-147. MR0144877

[2] P. J. Dukes and J. A. Short-Gershman (2012). A complete existence theory for Sarvate-Beam triple systems. Australas. J. Combin. 54, 261272. MR3013255

[3] K. O'Bryant (2004). A complete annotated bibliography of work related to Sidon sequences. Elec. J. Combin. \#DS11, 39pp.

[4] D. Sarvate and W. Beam (2007). A new type of block design. Bull. Inst. Combin. Appl. 50, 26-28. MR2317084

[5] R. M. Wilson (1975). An existence theory for pairwise balanced designs, III: Proof of the existence conjectures. J. Comb. Theory, Ser. A 18, 71-79. MR0366695

Peter J. Dukes

Mathematics and Statistics

UNIVERSITY OF VICTORIA

ViCTORIA, BC

CANADA

E-mail address: dukes@uvic.ca

JANE WodLinger

Mathematics and Statistics

UNIVERSITY OF VICTORIA

VICTORIA, BC

CANADA

E-mail address: jw@uvic.ca

Received December 5, 2012 